\title{
Topological Spin Excitations of Heisenberg Antiferromagnets in Two Dimensions
}

\author{
Tai Kai Ng \\ Department of Physics, Hong Kong University of Science and Technology, Kowloon, Hong Kong
}

(Received 14 December 1998)

\begin{abstract}
We discuss the construction and dynamics of vortexlike topological spin excitations in the Schwingerboson description of Heisenberg antiferromagnets in two dimensions. The topological spin excitations are Dirac fermions (with gap) when spin value $S$ is a half-integer. Experimental and theoretical implications of these excitations are being investigated. [S0031-9007(99)08982-6]

PACS numbers: 71.10.Fd, 75.10.Jm, 75.40.Gb
\end{abstract}

Recently, strong interest has been shown in the study of Heisenberg antiferromagnets on a two-dimensional square lattice. Early theoretical analysis showed that the low energy, long length scale behavior of the model should be described by the renormalized classical regime of the $(2+1) d \mathrm{O}(3)$ nonlinear sigma model $(\mathrm{NL} \sigma \mathrm{M})[1,2]$. The analysis was believed to be valid as long as the length scale under investigation was much larger than lattice spacing. More recently, in a series of experiments [3] and Monte Carlo simulations [4-6], it was discovered that the length scale at which the renormalized classical $\sigma$-model description becomes valid is surprisingly long ( $L \geq 200$ sites for $\operatorname{spin} S=1 / 2$ ) [5,6]. A quantum Monte Carlo study of the low energy spectrum of the model on finite size lattices also suggested that the spectrum in the $S=$ $1 / 2$ case disagrees rather strongly with the prediction of $\mathrm{NL} \sigma \mathrm{M}$ even when the size of the system $N$ is not too small $(N \leq 32)$ [7]. These anomalous findings suggest that contrary to usual beliefs, there may exist an intermediate energy/length scale where the behavior of the system deviates strongly from NL $\sigma \mathrm{M}$.

On the other hand, it has been shown by Read and Chakraborty [8] that fermionic spin excitations exist as topological excitations in the short-ranged resonant valence bond (RVB) phase of 2D $S=1 / 2$ Heisenberg model, and their analysis has been generalized [9] to the case (for arbitrary spin $S$ ) where the system is described by a Schwinger-boson mean-field theory (SBMFT) [10]. These topological excitations are finite energy excitations of 2D Heisenberg antiferromagnets that do not appear in conventional NL $\sigma \mathrm{M}$ description. However, the dynamics of the topological spin excitations were not investigated in those works $[8,9]$.

The purpose of this paper is to investigate, starting from SBMFT, the dynamics of the topological spin excitations constructed in Ref. [9] under some very general assumptions. In particular, we shall show that the existence of these topological excitations is consistent with the recently observed anomalous behaviors in the Heisenberg model. We shall also point out, in the case of $S=$ $1 / 2$ Heisenberg antiferromagnet, an interesting connection between the topological spin excitations and the spin ex- citations in the flux phase [11] of fermionic RVB meanfield theory.

We begin by reviewing briefly the construction of topological spin excitations in SBMFT of the 2D Heisenberg model. Following Ref. [9] we assume that the low energy physics of general spin- $S$ 2D Heisenberg model is described by the Schwinger-boson mean-field Hamiltonian

$$
\begin{aligned}
H_{\mathrm{MF}}=-J \sum_{i, j}[ & \Delta^{*}\left(Z_{i \uparrow} Z_{j \downarrow}-Z_{i \downarrow} Z_{j \uparrow}\right) \\
& \left.+ \text { H.c. }-|\Delta|^{2}\right]+\sum_{i \sigma} \lambda\left(\bar{Z}_{i \sigma} Z_{i \sigma}-2 S\right),
\end{aligned}
$$

where $\Delta\left(\Delta^{*}\right)$ and $\lambda$ are mean-field parameters determined by the mean-field equations $\Delta=\left\langle\left(Z_{i \uparrow} Z_{j \downarrow}-Z_{i \downarrow} Z_{j \uparrow}\right)\right\rangle$ and $\left\langle\bar{Z}_{i \uparrow} Z_{i \uparrow}\right\rangle+\left\langle\bar{Z}_{i \downarrow} Z_{i \downarrow}\right\rangle=2 S$. Notice that in two dimensions, long-ranged antiferromagnetic order exists at zero temperature corresponding to bose condensation $\langle Z\rangle \neq 0$ in SBMFT. We shall consider finite temperature $T \neq 0$ and $\langle Z\rangle=0$ in the following. (The effect of Bose condensation will be addressed at the end of this paper.) Notice that SBMFT offers a fairly accurate description of the low energy physics of a Heisenberg model at two dimensions [10], thus justifying the starting point of our theory.

To look for topological excitations in SBMFT, we notice that the structure of the mean-field theory resembles very much the Bardeen-Cooper-Schrieffer (BCS) theory for superconductivity, except that the spin pairs of bosons replace the electron (fermion) Cooper pairs in BCS theory. The resemblance of the two theories leads us to study vortex excitations in SBMFT, since vortices are stable topological excitations in BCS theory at two dimensions. In BCS theory, a vortex located at $\vec{r}=0$ is a solution of the BCS mean-field equation, where the order parameter $\Delta_{\mathrm{BCS}}(\vec{r})$ has a form $\Delta_{\mathrm{BCS}}(\vec{r})=f(r) e^{i \theta}$ in a polar coordinate, where $f(r)$ is real and positive. To minimize energy, a magnetic flux of $\pi$-flux quanta is trapped in the vortex core. The vortex solution in SBMFT has the same structure, except that the BCS order parameter $\Delta_{\mathrm{BCS}}$ is replaced by the Schwinger-boson order parameter $\Delta_{i j}$ and 
the vector potential $\vec{A}$ does not represent the physical magnetic field, but is a fictitious gauge field arising from phase fluctuations of order parameter $\Delta_{i j}[9,12]$. The existence and stability of the vortex solution was demonstrated in Ref. [9] in an effective Ginsburg-Landau theory. These vortex solutions are bosonic, $S=0$, topological excitations in SBMFT [9].

To construct topological spin excitations we note, that like vortices in superconductors where electronic bound states often exist inside the vortex core, boson bound states may exist inside vortices in SBMFT. In particular, we argued in Ref. [9] that, for a vortex centered at a lattice site, a bound state of $2 S$ bosons must be formed at the vortex center because of the constraint that there is always $2 S$ bosons per site in the Heisenberg model. In particular, because of statistics transmutation associated with binding quantum particles to a flux tube of $\pi$-flux quantum in two dimensions [13], the resulting excitation is a spin- $S$ fermion when $2 S$ is odd [9].

We shall now supply the mathematical details. An important difference between SBMFT and BCS theory is that the mean-field Hamiltonian (1) breaks the translational symmetry of the Heisenberg model by one lattice site. Correspondingly, there exist two lattice sites per unit cell in SBMFT and the fluctuations of the order parameter $\Delta_{i j}$ are described by two amplitude and two phase (uniform and staggered) fields in the continuum limit $[9,12]$, i.e.,

$$
\begin{aligned}
\Delta_{i, i \pm \nu}= & \frac{1}{2}\left[\phi\left(i \pm \frac{\nu}{2}\right)+q_{ \pm \nu}\left(i \pm \frac{\nu}{2}\right)\right] \\
& \times e^{i\left[\int^{i \pm \nu / 2} \vec{A} \cdot d \vec{x}+A_{ \pm \nu}^{s}(i \pm \nu / 2)\right]},
\end{aligned}
$$

where $q_{-\nu}=-q_{\nu}, A_{-\nu}^{s}=-A_{\nu}^{s}$ are "staggered" components of the amplitude and phase fluctuations of $\Delta$, respectively. $\phi$ and $\int^{x} \vec{A} \cdot d \vec{x}^{\prime}$ are the corresponding "uniform" components. The effective Ginsburg-Landau action for the continuum field variables is derived in Ref. [9] (see also Ref. [12]). We obtain to order $O\left(\mathrm{~m}^{0}\right)[\mathrm{m}$ is the mass gap for spin-wave excitations in SBMFT which is very small at low temperature $\left.\left(m \sim J e^{-J S / T}\right)\right], S_{\text {eff }}=$ $S_{u}+S_{s}$, where

$$
\begin{gathered}
S_{u} \sim \int d \tau \int d^{2} x\left\{\left[a-4\left(S+\frac{1}{2}\right)\right] \phi+\phi^{2}\right. \\
\left.+\left(S+\frac{1}{2}\right) \phi\left(\partial_{\mu} \theta-A_{\mu}\right)^{2}\right\}, \\
S_{s} \sim \int d \tau \int d^{2} x\left[\left(\frac{1}{2}-\frac{b}{\phi}\right)\left(q_{\mu}\right)^{2}+\frac{1}{2 e^{2}} F_{\mu \nu}^{2}\right. \\
\left.+i c F_{\mu \tau} q_{\mu}\right],
\end{gathered}
$$

where $e^{2} \sim m, a<4$, and $b$ and $c$ are constants of order $\mathrm{O}(1) . F_{\mu \nu}=\partial_{\mu} A_{\nu}^{s}-\partial_{\nu} A_{\mu}^{s}$ and $\theta / 2$ is the uniform phase field for the Schwinger bosons. Notice that the gauge symmetry of the uniform gauge field $\vec{A}$ is broken by $\left\langle\Delta_{i j}\right\rangle=\phi \neq 0$ in SBMFT. The existence of a stable vortex solution in SBMFT is tied with the $\vec{A}$ field as in the usual superconductors.

To derive vortex dynamics we concentrate at $S_{u}$ and introduce vortex 3 -current $\vec{j}^{v}$ in the phase field, $\partial_{\mu} \theta \rightarrow$ $\partial_{\mu} \theta+\partial_{\mu} \theta^{\prime}$, where $\theta^{\prime}$ is multivalued and $j_{\mu}^{v}=$ $\epsilon_{\mu \nu \lambda} \partial_{\nu} \partial_{\lambda} \theta^{\prime} \neq 0$. In the London limit where we treat $\phi$ as constant, a duality transformation can be performed where we can integrate out the $\theta$ field to obtain an effective action $S_{v}$ for vortices [14],

$$
\begin{aligned}
S_{v}=\int d \tau \int d^{2} x[ & \frac{1}{4 \phi}(\nabla \times \vec{a})^{2} \\
& \left.+i \vec{a} \cdot\left(\vec{j}^{v}-\nabla \times \vec{A}\right)\right]+S_{v}^{(0)},
\end{aligned}
$$

where $\nabla \times \vec{a} \sim\left(\nabla \theta^{\prime}-\vec{A}\right)$ and corresponds to the transverse part of the supercurrent field in superconductors. Notice that the amplitude field $\phi$ goes to zero at the center of the vortex. As a result, there exists an additional contribution to the vortex action, $S_{v}^{(0)} \sim\{$ (energy needed to create vortex core, $\epsilon_{v}$ ) $\times$ (length of vortex trajectory in space-time)\}. For $N$ vortices,

$$
S_{v}^{(0)} \sim \epsilon_{v} \sum_{i=1}^{N} \int d l_{i}=\epsilon_{v} \sum_{i=1}^{N} \int d \tau \sqrt{1+\frac{1}{c_{v}^{2}}}\left(\frac{d \vec{x}_{i}}{d \tau}\right)^{2},
$$

where $\vec{x}_{i}(\tau)$ represents the trajectory of the $i$ th vortex in Euclidean space-time, and $c_{v}$ is the limiting velocity. Notice that the suppression of the $\phi$ field at the vortex core also couples vortices to the staggered amplitude and phase fluctuations through the term $\left(-\frac{b}{\phi}\right) q_{\mu}^{2}$ in $S_{s}$ [9]. We shall first consider $S_{v}$ in the following.

The dynamics of a single vortex can be obtained by minimizing $S_{v}^{(0)}$ at real time. We find that $S_{v}^{(0)}$ describes relativistic particles with energy $E=\gamma \epsilon_{v}$, where $\gamma=$ $1 / \sqrt{1-\frac{\vec{v}^{2}}{c_{v}^{2}}}$ and $\vec{v}$ is the vortex velocity. In the absence of the $\nabla \times \vec{A}$ term, the particles have "charge" (vorticity) and interact with each other through an effective U(1) gauge field $\vec{a}$. For boson vortices the corresponding quantum field theory is a relativistic theory of scalar electrodynamics with charged bosons (vortices) [14]. In the presence of a trapped magnetic flux inside vortex core $\left(\nabla \times \vec{A}-\vec{j}_{v} \sim 0\right)$, the electric 3-current $\vec{j}_{v}$ is screened and the bosons decoupled from the gauge field $\vec{a}$. The resulting theory is a relativistic theory of bosons with short-ranged interactions, as is the case of vortices in usual superconductors [14].

To derive dynamics for the topological spin excitations we assume that, once the boson spins are bound to the vortex core, their spatial degree of freedom is quenched and the only modifications to the pure vortex action (4a) and (4b) are (1) the vortices now carry spin indices $m=-S,-S+1, \ldots, S$, and there are $2 S+1$ spin 
component of vortices, and (2) vortices become fermions when $2 S$ is odd. In particular, since the vortex action (4a) and (4b) is Lorentz invariant, the dynamics of the topological spin excitations must be described by $(2+1) d$-relativistic field theories of bosons when $S$ is an integer and by $(2+1) d$-relativistic field theories of fermions when $S$ is half-integer. To proceed further, we examine more carefully the symmetry constraints in SBMFT. The existence of two lattice sites per unit cell in SBMFT implies that there are also two quantum fields $\psi_{\sigma}^{A}$ and $\psi_{\sigma}^{B}$ in the continuum theory, representing spin- $\sigma$ vortices centered at $A$ - and $B$-sublattice sites, respectively [9]. Under reflection or rotation by $\pi / 2$ around the center of a square plaquette, the $A$ and $B$ sublattices are interchanged and, correspondingly, also $\psi_{\sigma}^{A}$ and $\psi_{\sigma}^{B}$. Notice that we have considered finite temperature where $\langle Z\rangle=0$ in our discussion and, correspondingly, parity (space-time reflection) symmetry is unbroken. In order to describe coupling of the bound boson spins at the vortex center to the fictitious gauge fields $\vec{A}$ and $\vec{A}_{s}$, the quantum fields $\psi_{\sigma}^{A}$ and $\psi_{\sigma}^{B}$ must also carry charge and are complex.

With these kinematics constraints, the quantum field theories for the topological spin excitations can be determined [15]. In the case of integer spins where the topological spin excitations are bosons, we construct quantum fields $\psi_{\sigma}^{+(-)}=\psi_{\sigma}^{A} \pm \psi_{\sigma}^{B}$ which are eigenstates of $\pi / 2$ rotation and reflection with eigenvalues \pm 1 . The dynamics of the $\psi_{\sigma}^{+(-)}$fields are described separately by relativistic theories of complex scalar fields. In the case of half-integer spins where the spin excitations are fermions, the corresponding theory which respects parity is a theory of Dirac fermions in $(2+1) d$ [15]. In the following we shall concentrate on the case of half-integer spin systems.

In this case, the topological spin excitations are Dirac spinors described by four-component spinor field $\psi_{\sigma}$, with

$$
\psi_{\sigma}(x)=\left(\begin{array}{l}
\psi_{\sigma}^{A}(x) \\
\psi_{\sigma}^{B}(x)
\end{array}\right), \quad \psi_{\sigma}^{A(B)}(x)=\left(\begin{array}{l}
\psi_{1 \sigma}^{A(B)}(x) \\
\psi_{2 \sigma}^{A(B)}(x)
\end{array}\right),
$$

where $\psi_{\sigma}^{A(B)}$ 's are two component fermion fields needed to describe positive and negative energy solutions of the Dirac equation. In terms of $\psi_{\sigma}$ the effective Lagrangian which transforms correctly under parity is

$$
\begin{aligned}
L_{\mathrm{eff}}= & \sum_{\sigma} \frac{i}{2}\left[\bar{\psi}_{\sigma} \gamma^{\mu}\left(\partial_{\mu} \psi_{\sigma}\right)-\left(\partial_{\mu} \bar{\psi}_{\sigma}\right) \gamma^{\mu} \psi_{\sigma}\right] \\
& -m \bar{\psi}_{\sigma} \psi_{\sigma}-\bar{\psi}_{\sigma} \gamma^{\mu} \psi_{\sigma} A_{\mu},
\end{aligned}
$$

where $\gamma^{\mu}$ 's are usual $4 \times 4$ Dirac matrices in $(2+$ 1) $d$ with $\mu=0,1,2 . \quad \vec{A}$ is the uniform gauge field. Notice that the staggered gauge field $\vec{A}_{s}$ decouples from the $\psi_{\sigma}$ field in this level, as can be checked easily from the transformation of $L_{\text {eff }}$ under staggered gauge transformation $\psi_{\sigma}^{A} \rightarrow \psi_{\sigma}^{A} e^{i \theta}$, and $\psi_{\sigma}^{B} \rightarrow \psi_{\sigma}^{B} e^{-i \theta}$. Notice also that the spin degrees of freedom $\sigma$ appear as internal degrees of freedom for the Dirac fermions.

It is interesting to compare the effective action $L_{\text {eff }}$ with the flux phase [11] of the fermionic mean-field theory of the Heisenberg model in the case $S=1 / 2$. In both cases, pockets of Dirac fermions describe the fermionic spin excitations at low energy. The number of species of Dirac fermions are the same in both cases - there are four "halfpockets" of Dirac fermions at about $\left(k_{x}, k_{y}\right)=\left( \pm \frac{\pi}{2}, \pm \frac{\pi}{2}\right)$ in the flux phase, and there are two "full-pockets" of Dirac fermions coming from two independent combinations of $\psi_{\sigma}^{A}$ and $\psi_{\sigma}^{B}$ in the present theory. The position of the "Dirac-pockets" in $\vec{k}$ space cannot be determined with certainty in our effective theory. Nevertheless, to make comparison with the flux phase we shall assume that the fermion pockets in our theory are centered around $\left(k_{x}, k_{y}\right)=\left( \pm \frac{\pi}{2}, \pm \frac{\pi}{2}\right)$. With this assumption the only difference between $L_{\text {eff }}$ and the flux phase is that there is a gap $\epsilon_{v}$ in the Dirac-fermion spectrum in our theory. In fact, in the limit $\epsilon_{v}=0$, it can be checked directly that $L_{\text {eff }}$ describes the continuum theory for the flux phase. The strong similarity between our effective theory and flux phase leads us to speculate that the flux phase in fact describes a new spin-disordered phase of 2D Heisenberg antiferromagnets, where antiferromagnetism is destroyed by driving the fermion mass gap $\epsilon_{v}$ to zero. We shall discuss this scenario in a future publication.

Next we consider the experimental consequences of the topological spin excitations. We shall first consider $S=1 / 2$ as an example. In terms of $\psi$, the spins carried by the Dirac fermions in the $S=1 / 2$ case are described by the operators

$$
\vec{S}^{A(B)}(x)=\frac{1}{2} \sum_{m, m^{\prime}} \sum_{k=1,2}\left[: \psi_{k m}^{+A(B)}(x) \vec{\sigma}_{m m^{\prime}} \psi_{k m^{\prime}}^{A(B)}(x):\right],
$$

where $\vec{S}^{A}(x)$ and $\vec{S}^{B}(x)$ are spin operators in the $A$ and $B$ sublattices, respectively, and $\vec{\sigma}$ are Pauli matrices, $m, m^{\prime}= \pm \frac{1}{2} .: \hat{O}:=\hat{O}-\langle\hat{O}\rangle_{G}$ are normal-ordered operators. The uniform and staggered spin operators can be constructed from $\vec{S}^{A(B)}$, where $\vec{m}(\vec{n})=\vec{S}^{A}+(-) \vec{S}^{B}$ describes spin fluctuations around momenta $\vec{q}=(0,0)$ and $\vec{q}=(\pi, \pi)$. Notice that, because of the constraint that there is one spin per site in the original Heisenberg model, the Dirac fermions together with the original boson spins must satisfy the constraint

$$
\sum_{\sigma}\left[\left(\sum_{k=1,2}: \psi_{k \sigma}^{+\alpha}(x) \psi_{k \sigma}^{\alpha}(x):\right)+\bar{Z}_{\sigma}^{\alpha}(x) Z_{\sigma}^{\alpha}(x)\right]=1,
$$

which introduces additional coupling between boson spins and topological spin excitations not included in $L_{\text {eff }}$.

Experimentally, the Dirac fermion spectrum can be observed directly in a neutron scattering experiment at energy $\omega>2 \epsilon_{v}$. Its contribution to the dynamic structure factor $S(\vec{q}, \omega)$ at $\vec{q} \sim(0,0)$ and $\vec{q} \sim(\pi, \pi)$ can be calculated directly using Eqs. (6) and (7). We find that the contributions are similar to $S(\vec{q}, \omega)$ calculated from flux phase, except that a gap $\sim 2 \epsilon_{v}$ is found in the spectral 
function. The existence of a Dirac-fermion spectrum also affects spin correlation at lower temperatures. To see that, we consider the antiferromagnetic spin-correlation length $\xi(T)$. In pure SBMFT, $\xi(T)$ is given at low temperature by [10]

$$
\xi(T)=\sqrt{2} \frac{\Delta J}{T} \exp \left(\frac{2 \pi \Delta J}{T} n_{B}\right),
$$

where $n_{B}$ is the density of bose-condensed spins in SBMFT at $T=0$. In the presence of the Dirac-fermion spectrum, the system gets more disordered at $T \neq 0$ because of the thermal effect associated with the extra degrees of freedom and $\xi(T)$ decreases. The effect can be estimated in a mean-field approximation using the constraint (8) [16], where the average number of Schwinger bosons per site $\left\langle n_{\mathrm{SB}}\right\rangle$ is given by

$$
\left\langle n_{\mathrm{SB}}\right\rangle=1-\sum_{k=1,2, \sigma}\left\langle: \psi_{k \sigma}^{+\alpha} \psi_{k \sigma}^{\alpha}:\right\rangle .
$$

It is straightforward to show that, at low temperature, $\left\langle n_{\mathrm{SB}}\right\rangle \sim 1-2 \exp \left(-\frac{\epsilon_{v}}{T}\right)$, and the leading correction at low temperature to $\xi(T)$ is obtained by replacing $n_{B} \rightarrow$ $n_{B}-2 \exp \left(-\frac{\epsilon_{v}}{T}\right)$ in Eq. (9). The reduction in $\xi(T)$ continues until $T \sim \epsilon_{v}$, when the number of thermally excited fermion spin excitations becomes large, and the spin correlation becomes qualitatively different from the prediction of SBMFT or NL $\sigma \mathrm{M}$. For general spin value $S, \epsilon_{v} \sim J S^{2}$ and the temperature at which the NL $\sigma \mathrm{M}$ description becomes invalid occurs at $T \sim J S^{2}$ which is deep inside the "quantum critical" regime for large values of $S$. For a small value of $S$, where there is no clear separation between energy scales $S J$ and $S^{2} J$, the whole quantum critical regime may be washed away by the existence of topological spin excitations. Such a scenario indeed seemed to be observed in Monte Carlo simulations of the $S=1 / 2$ 2D Heisenberg antiferromagnet [4-6], where the quantum critical regime predicted by the NL $\sigma \mathrm{M}$ description seems to be missing. Notice that for integer spin systems the contribution to dynamics structure factor $S(\vec{q}, \omega)$ from topological spin excitations is quite different from half-integer spin systems because of different statistics. However the effect on $\xi(T)$ should be qualitatively similar.

Finally we discuss the effects of gauge field $\vec{A}$. In the $T \rightarrow 0$ limit, where bose condensation of the boson spin takes place $(\langle Z\rangle \neq 0)$, the gauge field $\vec{A}$ becomes confining [17] and the topological spin excitations are confined in pairs by linear confining potential. At finite temperatures the confining potential is effective up to length scale $\sim$ correlation length $\xi(T)$, and the effect of confinement is expected to be strong at low temperature when the system is at the "renormalized classical" regime. As a result, the topological spin excitation spectrum at $\vec{q} \sim(0,0)$ is expected to be strongly modified from the free-Dirac-fermion prediction (for half-integer spins) at low temperature. However, the behavior of the topological spin-excitation spectrum at $\vec{q} \sim(\pi, \pi)$ is determined by the short-distance behaviors of spin pairs and should not be affected strongly by confinement. As a result, we expect that our predictions for $S(\vec{q}, \omega)$ at $\vec{q} \sim(\pi, \pi)$ and $\xi(T)$ remain qualitatively valid.

In summary, using SBMFT, we show in this paper that stable topological spin excitations exist in Heisenberg antiferromagnets at two dimensions. The topological spin excitations are described by relativistic quantum field theories of complex scalars when spin value $S$ is an integer, and they are Dirac fermions when $S$ is half-integer. We have discussed the theoretical and experimental consequences of these excitations and have pointed out that the existence of these spin excitations may be the reason for the anomalous results observed in recent experiments [3] and Monte Carlo simulations [4-7]. In particular, these excitations can be observed directly in neutron scattering experiments, and would provide a direct test of our theory.

The author thanks Professor N. Nagaosa and Professor P. A. Lee for many interesting discussions and also thanks the hospitality of the Aspen Center of Physics where part of this work was completed. This work is supported by HKRGC through Grant No. HKUST6143-97P.

[1] S. Chakravarty, B. I. Halperin, and D. R. Nelson, Phys. Rev. B 39, 2344 (1989); see also P. Hasenfratz and F. Niedermayer, Phys. Lett. B 268, 231 (1991).

[2] E. Manousakis, Rev. Mod. Phys. 63, 1 (1991).

[3] M. Greven et al., Phys. Rev. Lett. 72, 1096 (1994); Z. Phys. B 96, 465 (1995).

[4] N. Elstmer et al., Phys. Rev. Lett. 75, 938 (1995).

[5] B. B. Beard et al., Phys. Rev. Lett. 80, 1742 (1998).

[6] J-K. Kim and M. Troyer, Phys. Rev. Lett. 80, 2705 (1998).

[7] C. Lavalle et al., Phys. Rev. Lett. 80, 1746 (1998).

[8] N. Read and B. Chakraborty, Phys. Rev. B 40, 7133 (1989).

[9] T. K. Ng, Phys. Rev. B 52, 9491 (1995).

[10] D. P. Arovas and A. Auerbach, Phys. Rev. B 38, 316 (1988); see also D. Yoshioka, J. Phys. Soc. Jpn. 58, 1516 (1989).

[11] J. B. Marston and I. K. Affleck, Phys. Rev. B 39, 11538 (1989).

[12] N. Read and S. Sachdev, Phys. Rev. Lett. 62, 1694 (1989).

[13] F. Wilczek, Phys. Rev. Lett. 48, 1144 (1982).

[14] C. Dasgupta and B. I. Halperin, Phys. Rev. Lett. 47, 1556 (1981); M. P. A. Fisher and D. H. Lee, Phys. Rev. B 39, 2756 (1989).

[15] See, for example, S. Weinberg, The Quantum Theory of Fields I, (Cambridge University Press, Cambridge, England, 1995).

[16] See also T. K. Ng and C. H. Cheng, Phys. Rev. B 59, 6616 (1999) for an alternative approach.

[17] E. Fradkin and S. Shenker, 19, 3682 (1979). 